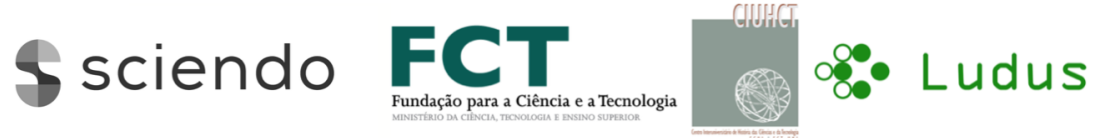

\section{The Five-Button Door Lock - Experiment And Discovery IN MATHEMATICS}

\author{
Shai Simonson and Timothy Woodcock
}

Stonehill College, North Easton, Massachusetts

\section{Introduction}

Experimentation, gathering data, and computation are an integral part of the process whereby mathematicians discover theorems and their proofs. Mathematics may be a science of exact proof, but the data and the process used in the discovery of certain proofs adds an experimental component. And, computers can help by creating data that reveal patterns. This productive methodology is one worth emphasizing at every level of mathematical instruction, but especially in primary, middle and high school curricula, where the emphasis on algorithms, methods, technique, and vocabulary leaves experiment and discovery as an afterthought.

Inspired by a question posted by Marc Dostie on the Rediscovering Mathematics Facebook page ${ }^{1}$, we consider a number of problems related to the following door lock. We offer this exploration as an example of how to incorporate discovery, experiment, and calculation into mathematics and pedagogy. An early version of this work was presented in an invited lecture at the Mathematics and Computer Science Colloquium at Providence College in $2015 .^{2}$

Programmable door locks such as the one in the figure, commonly found in schools, hospitals, and office buildings, provide a flexible way to maintain selective security and entry to different rooms and areas of buildings. To enter a room, a person presses certain buttons, then ENTER, and turns the handle. In this particular model of the lock, once a button is pressed, it cannot be pressed again, however, buttons can be pressed simultaneously, and the order in which the presses occur is significant.

\footnotetext{
${ }^{1}$ https://www.facebook.com/RediscoveringMath/

${ }^{2}$ The Five-Button Door Lock - Computation versus Mathematics, https://web.stonehill.edu/compsci/Shai_papers/Talk_PC.pptx
} 


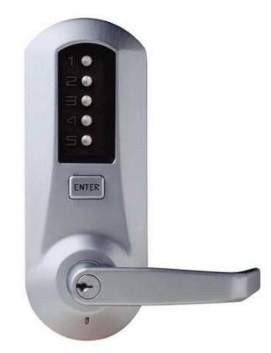

Figure 1: The Five Button Lock

These locks are called simplex locks. The number of combinations in simplex locks is related to Stirling numbers of the second kind and the Mahler basis for writing polynomials. Some details can be found in the work of Cuoco [Cuo05], McKay [McK16], and Velleman and Call [Vel95]. Cuoco, for example, has a nice result that the number of combinations that use all the buttons equals the number of combinations that use fewer than all the buttons. He uses this result to derive a recurrence very much like our main theorem in Section 4. Cuoco also points out that the number of combinations on an $m$-button lock that use all $m$ buttons and have $k$ presses is just the number of functions from an $m$-element set to a $k$-element set that "hit" each of the $k$ elements at least once, leading to a connection between the lock and polynomials written using the Mahler basis. Cuoco leaves many problems about the lock puzzle as exercises meant for further exploration.

McKay makes an explicit connection between the combinations in the lock puzzle and Stirling numbers of the second kind, focusing on partitioning an $n$-element set into $k$ non-empty subsets. Velleman and Call derive a connection between the locks and Eulerian numbers as well as the connection to Stirling numbers of the second kind. They derive asymptotic formulas and generalize the problem. Art Benjamin uses an exponential generating function to deduce an asymptotic formula [Ben96].

We examine the problem from scratch and stick to completely elementary methods. Our theme is to focus on using computers to help motivate proofs of theorems. Using a Java program and some generated data, we discover a number of new patterns and theorems about these locks and their combinations. We then prove these patterns are correct.

First, let's introduce a little terminology. In a simplex lock, a choice is a subset of $n$ numbers that are pressed simultaneously. There are $2^{n}$ choices in an $n$-button lock. For compactness and to more easily visualize the ordering of the choices, each subset is written as an ordered sequence of integers enclosed with parentheses, rather than with standard curly brackets and commas. For $n=5$, here are all the choices ordered 
lexicographically:

$$
\begin{aligned}
& (),(1),(12),(123),(1234),(12345),(1235),(124),(1245),(125),(13),(134),(1345),(135), \\
& (14),(145),(15),(2),(23),(234),(2345),(235),(24),(245),(25),(3),(34),(345),(35),(4),
\end{aligned}
$$

A combo is an ordered list of choices that contains at most one of each number from 1 to $n$.

After entering a combo, the ENTER key is pressed to open the lock and turn the handle. Each of the following three sequences represents a combo:

$$
(3)(2)(1)(5)(4) \quad(23)(5) \quad(531)(24)
$$

The first example has five choices, pressing the buttons $3,2,1,5,4$, in that order. The second example uses two choices, the first pressing 2 and 3 simultaneously, and then 5 alone; buttons 1 and 4 are not used in this combo. The last example also uses two choices: simultaneous pressing of 1,3 and 5 , followed by a simultaneous pressing of 2 and 4 . Note that in a given combo:

- Not all the buttons need be pressed. A blank combo is legal.

- No button may be pressed more than once. For example, (12)(32) is illegal.

- The order of the choices is significant. So for instance, $(12)(3)(45)$ is distinct from $(12)(45)(3)$.

- There is no implied order in any choice. The presses in any choice are simultaneous. That is, (135) is the same as (531), for example.

Marc Dostie wondered how long it would take, via brute force, to try all possible combinations and open the door. Indeed, he asked how many distinct combinations could be programmed for the five-button lock?

We answer his question for $n=5$, and provide various recurrence relations for the general $n$-button lock. We also derive some non-recursive formulas.

We begin by considering simpler versions of the lock in which the number of combinations is more clear. We then work slowly toward the actual lock in question adding complexity as we go. Working from special cases to the more general case is a well known way to help pinpoint the difficult aspects of a more general problem. 


\section{Variation I - $D(n)$ : No Order, No Simultaneous Presses}

In this variation, the order of the presses does not matter. The door opens simply by pressing the correct collection of buttons, in any order, without regard to simultaneous presses. Let $D(n)$ be the number of distinct combinations for such a lock with $n$ buttons. Then we immediately arrive at

Theorem 2.1. $D(n)=2^{n}$

Proof. Observe that $D(n)$ is equal to the number of subsets of $\{1,2, \ldots, n\}$, clearly; and it is well known that this number of subsets is equal to $2^{n}$. Equivalently, we have two choices for each of $n$ buttons, deciding whether it should be pressed or not.

We remark that a combination may be naturally represented as a binary string of length $n$, with a bit being associated to each button of the lock. A bit of value 1 indicates that its corresponding button should be pressed.

We also point out that $D(n)$ may be described through a simple recurrence relation, $D(n)=2 D(n-1)$ for positive $n$, with $D(0)=1$. Indeed, if $n \geqslant 1$, we may construct a combination for an $n$-button lock by first doing so for the first $n-1$ buttons, and then deciding whether the last button should be pressed or not. Regarding the base case, note that if we have a lock with no buttons, then there is one potential combination, in particular, where nothing is pressed.

We hope you agree that this variation on the lock is really quite easy.

\section{Variation II $-E(n)$ : Yes Order, No Simultaneous Presses}

In the second variation, we move closer to our actual problem by considering the order of the presses but not allowing any simultaneous presses. We define $E(n)$ to be the number of combinations for a lock of this type with $n$ buttons. Compared with $D(n)$, it is slightly harder to count $E(n)$. In doing so, we shall make use of the fairly-standard notation $P(n, k)$, denoting the number of permutations of $k$ distinct elements drawn from a pool of $n$ elements.

Theorem 3.1. $E(n)=\sum_{k=0}^{n} P(n, k)$

Proof. We partition the combinations according to how many buttons $k$ in total are pressed, where $k$ ranges from 0 to $n$. Obviously, for any given $k$, the number of potential combinations is $P(n, k)$. 
Through a standard exercise with the multiplication principle, we find that $P(n, k)=$ $n(n-1)(n-2) \cdots(n-k+1)$ for positive $k$, and thus

$$
E(n)=1+n+n(n-1)+n(n-1)(n-2)+\cdots+n !
$$

So for example,

$$
E(5)=1+5+5 \cdot 4+5 \cdot 4 \cdot 3+5 \cdot 4 \cdot 3 \cdot 2+5 \cdot 4 \cdot 3 \cdot 2 \cdot 1=326
$$

Now we also have

$$
E(n)=\sum_{k=0}^{n}\left(\begin{array}{l}
n \\
k
\end{array}\right) k !
$$

using an alternative closed form for $P(n, k)$. Indeed, to construct a combination using $k$ of the $n$ buttons, we may first select the ones that will be used, and then permute them. The chart below shows the first few values of $E(n)$ computed in the style of (3). Notice the emergence of Pascal's triangle in red, which arranges the coefficients $\left(\begin{array}{l}n \\ k\end{array}\right)$, being scaled by the respective factorial numbers in black:

\begin{tabular}{|c|c|c|}
\hline$n$ & $\sum\left(\begin{array}{l}n \\
k\end{array}\right) k !$ & $E(n)$ \\
\hline 0 & $1 \cdot 1$ & 1 \\
\hline 1 & $1 \cdot 1+1 \cdot 1$ & 2 \\
\hline 2 & $1 \cdot 1+2 \cdot 1+1 \cdot 2$ & 5 \\
\hline 3 & $1 \cdot 1+3 \cdot 1+3 \cdot 2+1 \cdot 6$ & 16 \\
\hline 4 & $1 \cdot 1+4 \cdot 1+6 \cdot 2+4 \cdot 6+1 \cdot 24$ & 65 \\
\hline 5 & $1 \cdot 1+5 \cdot 1+10 \cdot 2+10 \cdot 6+5 \cdot 24+1 \cdot 120$ & 326 \\
\hline
\end{tabular}

Though a recursive relationship for $E(n)$ is not immediately obvious, the chart does reveal a pattern, upon close inspection. Marking the values of $E(n)$ in blue, we observe that

$$
2=1 \cdot 1+1,5=2 \cdot 2+1,16=3 \cdot 5+1,65=4 \cdot 16+1,326=5 \cdot 65+1
$$

Therefore, we are led to

Theorem 3.2. For $n \geqslant 1, E(n)=n E(n-1)+1$

Proof. On the one hand, we may choose no buttons for our combination on an $n$-button lock, with one way to do so. On the other hand, to construct a general nonempty combination, we may select a first button, with $n$ choices, and then subsequently form any combination from the remaining $n-1$ buttons. 
The theorem might also have been discovered by looking at (2):

$$
E(5)=1+5[1+4+4 \cdot 3+4 \cdot 3 \cdot 2+4 \cdot 3 \cdot 2 \cdot 1]=1+5 E(4)
$$

And in general, for positive $n,(1)$ gives

$$
\begin{aligned}
E(n) & =1+n[1+(n-1)+(n-1)(n-2)+\cdots+(n-1) !] \\
& =1+n E(n-1)
\end{aligned}
$$

Also observe that the recurrence of Theorem 3.2, in connection with (3), yields that

$$
\sum_{k=0}^{n}\left(\begin{array}{l}
n \\
k
\end{array}\right) k !=\sum_{k=0}^{n-1} n\left(\begin{array}{c}
n-1 \\
k
\end{array}\right) k !+1=\sum_{k=1}^{n} n\left(\begin{array}{l}
n-1 \\
k-1
\end{array}\right)(k-1) !+1
$$

Hence, noting that $\left(\begin{array}{l}n \\ 0\end{array}\right) \cdot 0 !=1$, we naturally conjecture that the remaining terms in the sum on the left match up with those of the sum on the right. That is to say, we expect

Theorem 3.3. For $1 \leqslant k \leqslant n$,

$$
\left(\begin{array}{l}
n \\
k
\end{array}\right) k !=n\left(\begin{array}{l}
n-1 \\
k-1
\end{array}\right)(k-1) !
$$

Proof. Appealing to the well-known formula $\left(\begin{array}{l}n \\ k\end{array}\right)=\frac{n !}{k !(n-k) !}$, we realize that both sides of the equality in the theorem reduce to $\frac{n !}{(n-k) !}$.

Alternatively, the theorem could have been proved by observing that both of its expressions are equal to $P(n, k)$. In particular, $n\left(\begin{array}{c}n-1 \\ k-1\end{array}\right)(k-1) !=n P(n-1, k-1)$. And, constructing a nonempty combination of $k$ buttons by first choosing its first button (just as we did in the proof of Theorem 3.2), we see that the number of $k$-button possibilities, $P(n, k)$, is also equal to $n P(n-1, k-1)$.

With some motivating success on the simpler variations, we finally move on to the original problem. The original problem is a much harder problem, and will require more investigating and experimenting.

\section{Variation III $-F(n)$ : Yes Order, Yes Simultaneous Presses}

This variation is the simplex lock in its general form. Let $F(n)$ be the number of combos in an $n$-button simplex lock.

With no clear plan of how to calculate $F(5)$ mathematically, we take a programmer's point of view, and construct an algorithm to generate combos in an organized fashion. 
Our program is a natural way to generate combos, guaranteeing that no combo is counted twice or omitted. We may not yet understand what is going on, but at least we will know the value of $F(5)$, and hopefully, we will also generate some useful data and patterns.

Algorithm for Generating Combos:

1. Start with the blank combo.

2. While possible

// Generate the next combo from the previous one

a. If numbers 1-5 are not all included, append the next choice that does not duplicate a number. (We use the lexicographic ordering of choices described earlier.)

b. If all 5 numbers are included, then remove the rightmost choice from the combo, and replace it with the next smallest choice that does not duplicate a number in the combo nor repeat a previously generated combo.

We programmed this algorithm in Java. See Appendix.

And, here is the answer for Mr. Dostie: 1082 combos for a 5-button lock. In other words, $F(5)=1082$. The combinations are listed in order from top to bottom in five columns in the Appendix.

Of course, this list is not very satisfying in comparison to the more general results we have for variations I and II, $D(n)$ and $E(n)$. Moreover, the data below seems way too big and disorganized to notice any patterns, so for now, any recurrence equations or closed form for $F(n)$ is elusive.

In order to learn something about the nature of these combos, we examine the combos tabulated by our algorithm for 0 to 4 button locks.

\section{Buttons Choices Combos}

$\begin{array}{ccc}0 & () & \text { One Combo } \\ & ()(1) & \text { Two Combos } \\ 1 & () & (1) \\ & & (\text { Six Combos }\end{array}$




\section{The Five-Button Door Lock - Experiment and Discovery in Mathematics}

$(2)(1)$
3
() (1) (12) (123) (13) (2) (23) (3)
26 Combos

()
(1)
(1) (2)
(1) (2) (3)
(1) (23)
(1) (3)
$(1)(3)(2)$

$(12)$
$(12)(3)$
$(123)$
$(13)$
$(13)(2)$

(2)

(2) (1)

(2) (1) (3)

(2) (13)

(2) (3)

(2) (3) (1)

(23)

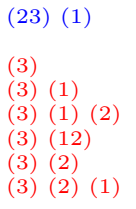

It seems that:

$$
\begin{aligned}
F(3) & =\left(\begin{array}{l}
3 \\
0
\end{array}\right)+\left(\begin{array}{l}
3 \\
1
\end{array}\right) F(2)+\left(\begin{array}{l}
3 \\
2
\end{array}\right) F(1)+\left(\begin{array}{l}
3 \\
3
\end{array}\right) F(0) \\
& =1+3(6)+3(2)+1(1)=26
\end{aligned}
$$

Now let's look at 4 buttons. The choices are:

$$
()(1)(12)(123)(1234)(124)(13)(134)(14)(2)(23)(234)(24)(3)(34)(4)
$$

and there are 150 Combos. The colors reveal the recursive structure in the formula.

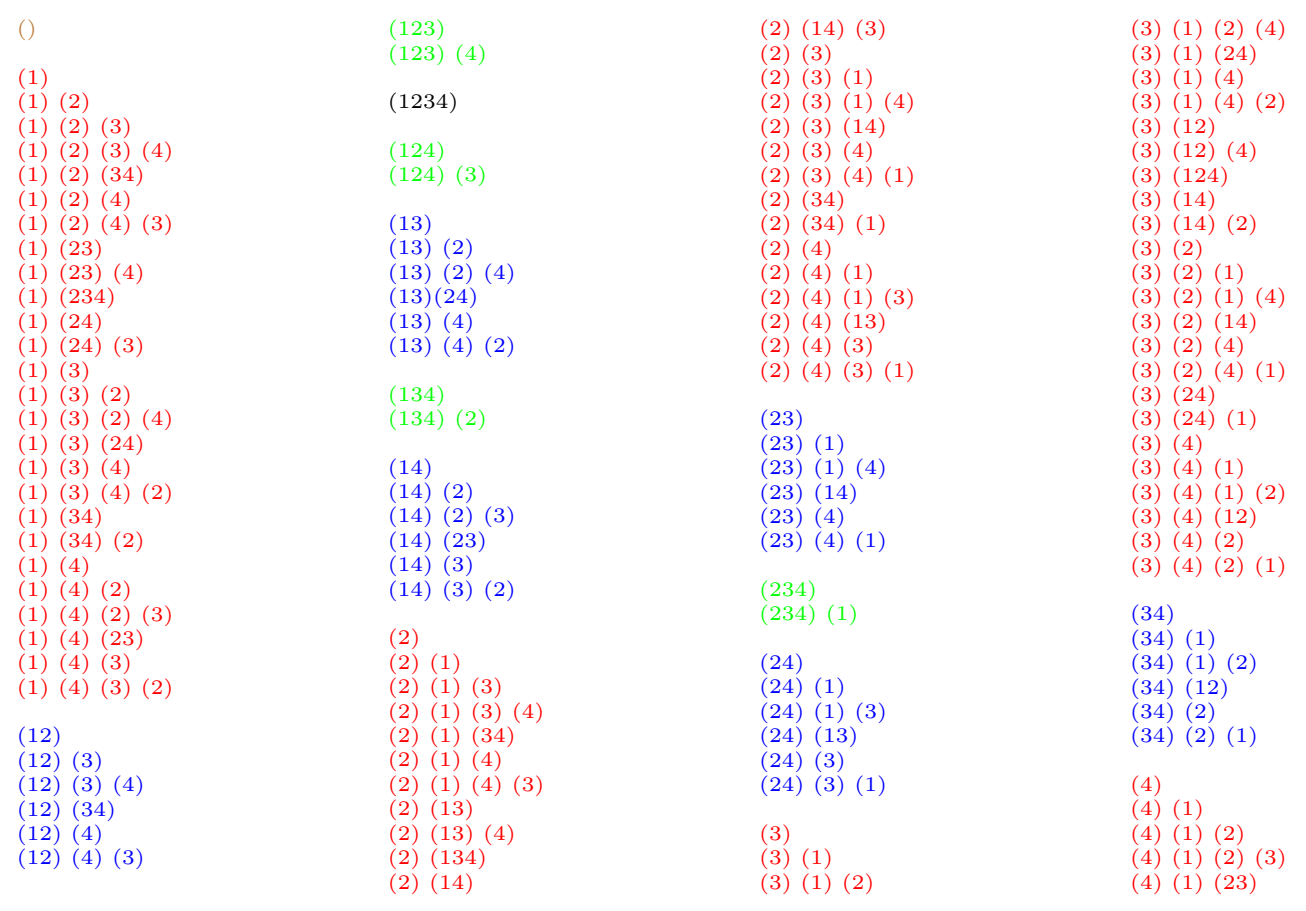




$\begin{array}{llll}(4)(1)(3) & (4)(13)(2) & (4)(2)(3)(1) & (4)(3)(12) \\ (4)(1)(3)(2) & (4)(2) & (4)(23) & (4)(3)(2) \\ (4)(12) & (4)(2)(1) & (4)(3) & (1) \\ (4)(12)(3) & (4)(2)(1)(3) & (4)(3)(1) & (4)(3)(2)(1) \\ (4)(123) & (4)(2)(13) & (4)(3)(1)(2) & \end{array}$

Notice that

$$
\begin{aligned}
F(4) & =\left(\begin{array}{l}
4 \\
0
\end{array}\right)+\left(\begin{array}{l}
4 \\
1
\end{array}\right) F(3)+\left(\begin{array}{l}
4 \\
2
\end{array}\right) F(2)+\left(\begin{array}{l}
4 \\
3
\end{array}\right) F(1)+\left(\begin{array}{l}
4 \\
4
\end{array}\right) F(0) \\
& =1+4(26)+6(6)+4(2)+1(1)=150
\end{aligned}
$$

Now we are getting somewhere. The data for $n=1$ and $n=2$ may not be so revealing because it is too small, but the data for $n=3$ and $n=4$ is mighty helpful. We colored the various combos to emphasize the pattern that emerges. And, this time, the patterns not only reveal the recursive structure, but also suggest a proof.

It seems that in general:

\section{Theorem 4.1.}

$$
F(n)=\left(\begin{array}{l}
n \\
0
\end{array}\right)+\sum_{k=1}^{n}\left(\begin{array}{l}
n \\
k
\end{array}\right) F(n-k)
$$

Proof. Either we select some buttons or we select none. There is one way to select no buttons, hence the term of $\left(\begin{array}{l}n \\ 0\end{array}\right)$ in the formula. Otherwise, select which buttons will be in the first subset, i.e. in the first choice. The number $k$ of these buttons ranges from 1 to $n$; and there are $\left(\begin{array}{l}n \\ k\end{array}\right)$ ways to choose $k$ of $n$ buttons for the first subset. This leaves us with a lock of $n-k$ buttons, for our subsequent choices.

And thus, for $n=5$, the number of blank combos is $\left(\begin{array}{l}5 \\ 0\end{array}\right)$, equal to 1 . And there are:

- $\left(\begin{array}{l}5 \\ 1\end{array}\right)$ ways to choose 1 button followed by a 4-button lock.

- $\left(\begin{array}{l}5 \\ 2\end{array}\right)$ ways to choose 2 buttons followed by a 3-button lock.

- $\left(\begin{array}{l}5 \\ 3\end{array}\right)$ ways to choose 3 buttons followed by a 2-button lock.

- $\left(\begin{array}{l}5 \\ 4\end{array}\right)$ ways to choose 4 buttons followed by a 1-button lock.

- $\left(\begin{array}{l}5 \\ 5\end{array}\right)$ ways to choose 5 buttons followed by a 0-button lock.

Therefore,

$$
\begin{aligned}
F(5) & =\left(\begin{array}{l}
5 \\
0
\end{array}\right)+\left(\begin{array}{l}
5 \\
1
\end{array}\right) F(4)+\left(\begin{array}{l}
5 \\
2
\end{array}\right) F(3)+\left(\begin{array}{l}
5 \\
3
\end{array}\right) F(2)+\left(\begin{array}{l}
5 \\
4
\end{array}\right) F(1)+\left(\begin{array}{l}
5 \\
5
\end{array}\right) F(0) \\
& =1+5(150)+10(26)+10(6)+5(2)+1(1)
\end{aligned}
$$

Here is what we have discovered so far: 


\begin{tabular}{|l|l|r|}
$n$ & & $F(n)$ \\
\hline 0 & 1 & 1 \\
1 & $1+1(1)$ & 2 \\
2 & $1+2(2)+1(1)$ & 6 \\
3 & $1+3(6)+3(2)+1(1)$ & 26 \\
4 & $1+4(26)+6(6)+4(2)+1(1)$ & 150 \\
5 & $1+5(150)+10(26)+10(6)+5(2)+1(1)$ & 1082
\end{tabular} \mid

In the middle column, notice the red Pascal's triangle and the black values of $F(n)$ for $n<5$.

Let's investigate the $n=5$ case more deeply, and count the combos in a non-recursive manner. For each number $k$ of buttons pressed, in total, the number of combos is $\left(\begin{array}{l}5 \\ k\end{array}\right)$ times the number of ways to press the $k$ buttons in some order with simultaneous presses allowed.

A step is any single, possibly simultaneous, pressing of button(s). Partitioning the cases over the total number of buttons pressed, and the total number of steps, we get, for our respective numbers of combo possibilities on a 5 -button lock:

- 0 buttons: $\left(\begin{array}{l}5 \\ 0\end{array}\right)(1)=1$

- 1 button: $\left(\begin{array}{l}5 \\ 1\end{array}\right)(1)=5$

- 2 buttons: $\left(\begin{array}{l}5 \\ 2\end{array}\right)(1+2)=10+20=30$

- There is one way to have one step, and two ways to have two steps. Either you press both buttons at once (1 possibility) or each separately (2 possibilities, because order matters).

- 3 buttons: $\left(\begin{array}{l}5 \\ 3\end{array}\right)(1+6+6)=10+60+60=130$

- There is one way to have one step, six ways to have two steps, and six ways to have three steps. Either you press all three buttons at once (1 possibility), or split them $2-1$ or $1-2$ (for $3+3$ possibilities, noting that $\left(\begin{array}{l}3 \\ 2\end{array}\right)=3$ ), or press all three separately (for $3 !=6$ possibilities).

- 4 buttons: $\left(\begin{array}{l}5 \\ 4\end{array}\right)(1+14+36+24)=5+70+180+120=375$

- There is one way to have one step, 14 ways to have two steps, 36 ways to have three steps, and 24 ways to have 4 steps. Either you press all the buttons at once (1), split $1-3$ or $3-1$ or $2-2(4+4+6)$, or split $1-1-2$ or $1-2-1$ or $2-1-1$ $(12 \cdot 3)$, or press all four separately $(24)$. 
- 5 buttons: $\left(\begin{array}{l}5 \\ 5\end{array}\right)(1+30+150+240+120)=541$

- There is one way to have one step, 30 ways to have two steps, 150 ways to have three steps, 240 ways to have four steps, and 120 ways to have five steps. Either you press all the buttons at once (1), split 1-4 or 4-1 or 2-3 or $3-2(5+5+10+10)$, split $1-1-3$ or $1-3-1$ or $3-1-1$ or $1-2-2$ or $2-1-2$ or $2-2-1$ $(20+20+20+30+30+30)$, or split $1-1-1-2$ or $1-2-1-1$ or $1-1-2-1$ or $1-1-1-2$ $(60 \cdot 4)$, or press all five separately $(120)$.

Summing over our cases, we see that the total number of combos equals

$$
1+5+30+130+375+541=1082
$$

A similar analysis can be made for locks with smaller numbers of buttons. A summary is shown in the tables below for $n$ equal 2 through 5 .

$$
\mathbf{n}=\mathbf{2}
$$

\begin{tabular}{|l|ccrr|}
\hline Pressed & 0 & 1 & 2 & Total \\
\hline 0 & 1 & 0 & 0 & 1 \\
\hline 1 & 0 & 2 & 0 & 2 \\
\hline 2 & 0 & 1 & 2 & 3 \\
\hline Total & 1 & 3 & 2 & $\mathbf{6}$ \\
\hline
\end{tabular}

$$
\mathbf{n}=\mathbf{3}
$$

\begin{tabular}{|l|rrrrr|}
\hline Pressed Steps & 0 & 1 & 2 & 3 & Total \\
\hline 0 & 1 & 0 & 0 & 0 & 1 \\
\hline 1 & 0 & 3 & 0 & 0 & 3 \\
\hline 2 & 0 & 3 & 6 & 0 & 9 \\
\hline 3 & 0 & 1 & 6 & 6 & 13 \\
\hline Total & 1 & 1 & 12 & 6 & $\mathbf{2 6}$ \\
\hline
\end{tabular}

$$
\mathbf{n}=\mathbf{4}
$$

\begin{tabular}{|l|rrrrrr|}
\hline Pressed Steps & 0 & 1 & 2 & 3 & 4 & Total \\
\hline 0 & 1 & 0 & 0 & 0 & 0 & 1 \\
\hline 1 & 0 & 4 & 0 & 0 & 0 & 4 \\
\hline 2 & 0 & 6 & 12 & 0 & 0 & 18 \\
\hline 3 & 0 & 4 & 24 & 24 & 0 & 52 \\
\hline 4 & 0 & 1 & 14 & 36 & 24 & 75 \\
\hline Total & 1 & 15 & 50 & 60 & 24 & $\mathbf{1 5 0}$ \\
\hline
\end{tabular}




$$
\mathbf{n}=5
$$

\begin{tabular}{|l|rrrrrrr|}
\hline Pressed Steps & 0 & 1 & 2 & 3 & 4 & 5 & Total \\
\hline 0 & 1 & 0 & 0 & 0 & 0 & 0 & 1 \\
\hline 1 & 0 & 5 & 0 & 0 & 0 & 0 & 5 \\
\hline 2 & 0 & 10 & 20 & 0 & 0 & 0 & 30 \\
\hline 3 & 0 & 10 & 60 & 60 & 0 & 0 & 130 \\
\hline 4 & 0 & 5 & 70 & 180 & 120 & 0 & 375 \\
\hline 5 & 0 & 1 & 30 & 150 & 240 & 120 & 541 \\
\hline Total & 1 & 31 & 180 & 390 & 360 & 120 & $\mathbf{1 0 8 2}$ \\
\hline
\end{tabular}

These tables built without any recursive idea, nonetheless, display some unexpected recursive structure.

Definition 1. Let $f(n, k)$ be the number of combos in an $n$-button lock using $k$ steps.

Regarding our definition, we remark that $f(n, 0)=1, f(0, k)=0$, for positive $k$, and $f(n, k)=0$, for $n<k$.

\section{Theorem 4.2.}

$$
f(n, k)=(k+1) f(n-1, k)+k f(n-1, k-1)
$$

Proof. For $n$ and $k$ greater than 0, we observe that the number of $k$-step combinations that do not use the $n$-th button is $f(n-1, k)$. The number of combos that use the $n$-th button in a step with at least one other button is $k f(n-1, k)$; for we have $k$ choices in deciding which step the last button should join, given a $k$-step combination with $n-1$ buttons. And finally, the number of $k$-step combinations with $n$ buttons where the $n$-th button is a step on its own is $k f(n-1, k-1)$; given a $(k-1)$-step combination with $n-1$ buttons, we choose a position in the step sequence to insert the individual step of the $n$-th button, with $k$ options (at the very front, or the very end, or in between any pair of steps along the way).

The table below shows the values of $f(n, k)$ for $n$ equal 0 to 5 , and $k$ equal 0 to $\mathrm{n}$. 
$\mathbf{f}(\mathbf{n}, \mathbf{k})$

\begin{tabular}{|c|c|c|c|c|c|c|c|}
\hline$n$ & 0 & 1 & 2 & 3 & 4 & 5 & Total $=F(n)$ \\
\hline 0 & 1 & 0 & 0 & 0 & 0 & 0 & 1 \\
\hline 1 & 1 & 1 & 0 & 0 & 0 & 0 & 2 \\
\hline 2 & 1 & 3 & 2 & 0 & 0 & 0 & 6 \\
\hline 3 & 1 & 7 & 12 & 6 & 0 & 0 & 26 \\
\hline 4 & 1 & 15 & 50 & 60 & 24 & 0 & 150 \\
\hline 5 & 1 & 31 & 180 & 390 & 360 & 120 & 1082 \\
\hline
\end{tabular}

For example, let us consider $f(5,4)$. Either we use button 5 or we do not. Without button 5 we have $f(4,4)=24$. With button 5 , we can either include the button in a step with other buttons or leave it on its own. If we include button 5 in a step with other buttons, we choose which of 4 steps presses 5 , giving $4 f(4,4)=4(24)$. Leaving button 5 on its own, we choose which one of 4 slots gets button 5 , and we have $4 f(4,3)=4(60)$. So $f(5,4)=5(24)+4(60)=360$.

\section{An Inclusion-Exclusion Approach toward Closed Forms for $f(n, k)$ and $F(n)$}

We still do not have a closed form for $f(n, k)$ or $F(n)$. However, examining the $f(n, k)$ table with more scrutiny reveals a non-recursive way to formulate $f(n, k)$, and in turn $F(n)$, using the principle of inclusion-exclusion.

\section{Theorem 5.1.}

$$
f(n, k)=\left(\begin{array}{l}
k \\
0
\end{array}\right)(k+1)^{n}-\left(\begin{array}{l}
k \\
1
\end{array}\right) k^{n}+\left(\begin{array}{l}
k \\
2
\end{array}\right)(k-1)^{n}-\cdots \pm\left(\begin{array}{l}
k \\
k
\end{array}\right) 1^{n}
$$

Proof. We observe that a $k$-choice combination on an $n$-button lock, with buttons labeled $1,2, \ldots, n$, corresponds naturally to a sequence $\left(a_{1}, \ldots, a_{n}\right)$ over $0,1,2, \ldots, k$ with $a_{m}$ indicating the choice number in which button $m$ appears, where we impose the restriction that each element of $1,2, \ldots, k$ appears at least once in the sequence. Note that $a_{m}=0$ means that button $m$ is not used in the combination.

Let $X$ be the set of all $n$-tuples over $0,1,2, \ldots, k$, and for $m$ in $1,2, \ldots, k$, let $Y_{m}$ be the subset of all elements of $X$ that are missing $m$ as a term. Then the set of all legal $k$-choice combinations on an $n$-button lock is

$$
X-\left(Y_{1} \cup Y_{2} \cup \cdots \cup Y_{k}\right)
$$


By the principle of inclusion-exclusion, the cardinality of this set is:

$$
|X|-\left(\left|Y_{1}\right|+\cdots+\left|Y_{k}\right|\right)+\left(\left|Y_{1} Y_{2}\right|+\left|Y_{1} Y_{3}\right|+\cdots\right)-(\cdots
$$

And this amounts to:

$$
(k+1)^{n}-\left(\begin{array}{l}
k \\
1
\end{array}\right) k^{n}+\left(\begin{array}{l}
k \\
2
\end{array}\right)(k-1)^{n}-\cdots
$$

For example,

$$
\begin{gathered}
f(5,2)=3^{5}-2\left(2^{5}\right)+1=180 \\
f(5,3)=4^{5}-3\left(3^{5}\right)+3\left(2^{5}\right)-1=390
\end{gathered}
$$

To obtain the total number of combos, $F(n)$, we sum $f(n, k)$ as $k$ runs from 0 to $n$. After collecting terms, we have:

$$
\begin{aligned}
F(n)= & (n+1)^{n} \\
& -n^{n}\left[\left(\begin{array}{l}
n \\
1
\end{array}\right)-\left(\begin{array}{c}
n-1 \\
0
\end{array}\right)\right] \\
& +(n-1)^{n}\left[\left(\begin{array}{l}
n \\
2
\end{array}\right)-\left(\begin{array}{c}
n-1 \\
1
\end{array}\right)+\left(\begin{array}{c}
n-2 \\
0
\end{array}\right)\right] \\
& -(n-2)^{n}\left[\left(\begin{array}{l}
n \\
3
\end{array}\right)-\left(\begin{array}{c}
n-1 \\
2
\end{array}\right)+\left(\begin{array}{c}
n-2 \\
1
\end{array}\right)-\left(\begin{array}{c}
n-3 \\
0
\end{array}\right)\right] \\
& +\cdots
\end{aligned}
$$


For example,

$$
\begin{aligned}
F(4)= & 5^{4} \\
& -4^{4}\left[\left(\begin{array}{l}
4 \\
1
\end{array}\right)-\left(\begin{array}{l}
3 \\
0
\end{array}\right)\right] \\
& +3^{4}\left[\left(\begin{array}{l}
4 \\
2
\end{array}\right)-\left(\begin{array}{l}
3 \\
1
\end{array}\right)+\left(\begin{array}{l}
2 \\
0
\end{array}\right)\right] \\
& -2^{4}\left[\left(\begin{array}{l}
4 \\
3
\end{array}\right)-\left(\begin{array}{l}
3 \\
2
\end{array}\right)+\left(\begin{array}{l}
2 \\
1
\end{array}\right)-\left(\begin{array}{l}
1 \\
0
\end{array}\right)\right] \\
& +1^{4}\left[\left(\begin{array}{l}
4 \\
4
\end{array}\right)-\left(\begin{array}{l}
3 \\
3
\end{array}\right)+\left(\begin{array}{l}
2 \\
2
\end{array}\right)-\left(\begin{array}{l}
1 \\
1
\end{array}\right)+\left(\begin{array}{l}
0 \\
0
\end{array}\right)\right] \\
= & 150
\end{aligned}
$$

\section{Generalizing the Triangle Numbers}

With our success with $f(n, k)$, we define $g(n, k)$ to be the number of combinations on an $n$-button lock, consisting of $k$ choices, but where the order of the choices is not considered relevant. This is a simpler problem than the original one we considered, but it has a beautiful recursive structure that can be considered a generalization of the triangle numbers. Indeed, we show that $g(n, n-1)$ is the $n$-th triangle number.

First note that $f(n, k)=k ! g(n, k)$. Similar to the recurrence relation

$$
f(n, k)=(k+1) f(n-1, k)+k f(n-1, k-1)
$$

we have

\section{Theorem 6.1.}

$$
g(n, k)=(k+1) g(n-1, k)+g(n-1, k-1)
$$

Proof. Similar to the proof for $f(n, k)$.

Theorem 6.2. $g(n, k)$ is equal to the sum of all terms $2^{a_{2}} 3^{a_{3}}(k+1)^{a_{k+1}}$, where the individual exponents $a_{i}$ are nonnegative integers, and the total exponent is at most $n-k$.

Proof. Using the recurrence $g(n, k)=(k+1) g(n-1, k)+g(n-1, k-1)$, with base cases $g(n, 0)=1$ for all $n$, the result follows by examining a Pascal-like triangle for arranging all of the $g(n, k)$. 
For example, to compute $g(5,3)$, we observe that 1 is the only term with total exponent zero among the sum of all $2^{a_{2}} 3^{a_{3}} 4^{a_{4}}$. Next, we notice that the sum of terms with total exponent 1 is $2+3+4=9$. Finally, the sum of terms with total exponent 2 is $2(2+3+4)+3(3+4)+4(4)=55$. So in total, $g(5,3)=1+9+55=65$.

This implies that $g(n, n-1)$ is the sum of all terms $2^{a_{2}} 3^{a_{3}} n^{a_{n}}$, where the total exponent is at most 1 . In other words, $g(n, n-1)$ is the $n$-th triangular number.

\section{Conclusions and Open Questions}

We solved the problem asked by Marc Dostie and explored some variations, providing an example of elementary mathematical research motivated by exploration and experiment. With the help of a computer program, we found a beautiful recursive structure for $F(n)$, and by inventing $f(n, k)$, came up with some nice non-recursive formulas for both $F(n)$ and $f(n, k)$. It would be very interesting to find simpler closed forms for $F(n)$ and $f(n, k)$, that make no use of explicit summations.

\section{References}

[Ben96] Benjamin, Art. "Combinatorics and campus security." Journal for Undergraduate Mathematics and its Applications, 17(2), 1996: 111-116.

to the list of references.

[Cuo05] Al Cuoco. Mathematical Connections, A Companion for Teachers and Others. MAA, 2005, 173-184.

[McK16] Christopher M. McKay. "On the n-Button Lock Problem and Combinatorial Proofs to Fibonacci Identities." Bachelor's Thesis in Mathematics, Stonehill College, 2016.

[Vel95] Daniel J. Velleman and Gregory S. Call. "Permutations and Combination Locks." Mathematics Magazine 68:4, 1995: 243-253. 


\section{A Appendix}

The 1082 Combos for the 5-Button Lock and the Java Program

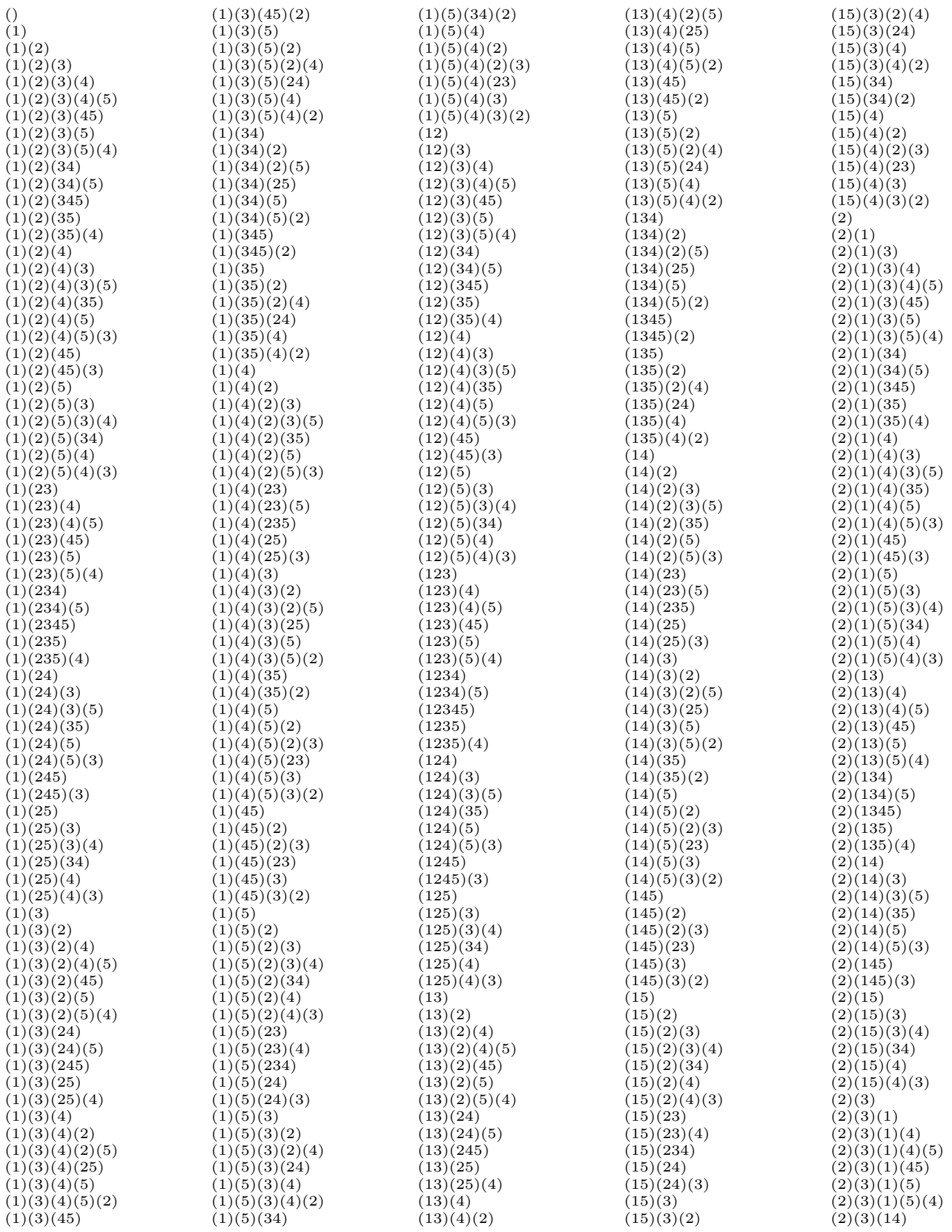




\section{The Five-Button Door Lock - Experiment and Discovery in Mathematics}

\begin{tabular}{|c|c|c|c|c|}
\hline$(2)(3)(14)(5)$ & $(2)(5)(3)(1)(4)$ & $(245)$ & (3)(15) & $(3)(5)$ \\
\hline$(2)(3)(145)$ & $(2)(5)(3)(14)$ & $(245)(1)$ & $(3)(15)(2)$ & $(3)(5)(1)$ \\
\hline$(2)(3)(15)$ & $(2)(5)(3)(4)$ & $(245)(1)(3)$ & $(3)(15)(2)(4)$ & $(3)(5)(1)(2)$ \\
\hline$(2)(3)(15)(4)$ & $(2)(5)(3)(4)(1)$ & $(245)(13)$ & $(3)(15)(24)$ & $(3)(5)(1)(2)(4)$ \\
\hline$(2)(3)(4)$ & $(2)(5)(34)$ & $(245)(3)$ & (3) (15)(4) & $(3)(5)(1)(24)$ \\
\hline$(2)(3)(4)(1)$ & $(2)(5)(34)(1)$ & $(245)(3)(1)$ & $(3)(15)(4)(2)$ & $(3)(5)(1)(4)$ \\
\hline$(2)(3)(4)(1)(5)$ & $(2)(5)(4)$ & $(25)$ & (3) (2) & $(3)(5)(1)(4)(2)$ \\
\hline$(2)(3)(4)(15)$ & $(2)(5)(4)(1)$ & $(25)(1)$ & $(3)(2)(1)$ & (3) (5)(12) \\
\hline$(2)(3)(4)(5)$ & $(2)(5)(4)(1)(3)$ & $(25)(1)(3)$ & $(3)(2)(1)(4)$ & $(3)(5)(12)(4)$ \\
\hline$(2)(3)(4)(5)(1)$ & $(2)(5)(4)(13)$ & $(25)(1)(3)(4)$ & $(3)(2)(1)(4)(5)$ & $(3)(5)(124)$ \\
\hline$(2)(3)(45)$ & $(2)(5)(4)(3)$ & $(25)(1)(34)$ & $(3)(2)(1)(45)$ & (3) (5)(14) \\
\hline$(2)(3)(45)(1)$ & $(2)(5)(4)(3)(1)$ & $(25)(1)(4)$ & $(3)(2)(1)(5)$ & $(3)(5)(14)(2)$ \\
\hline$(2)(3)(5)$ & (23) & $(25)(1)(4)(3)$ & $(3)(2)(1)(5)(4)$ & $(3)(5)(2)$ \\
\hline$(2)(3)(5)(1)$ & $(23)(1)$ & $(25)(13)$ & $(3)(2)(14)$ & $(3)(5)(2)(1)$ \\
\hline$(2)(3)(5)(1)(4)$ & $(23)(1)(4)$ & $(25)(13)(4)$ & $(3)(2)(14)(5)$ & $(3)(5)(2)(1)(4)$ \\
\hline$(2)(3)(5)(14)$ & $(23)(1)(4)(5)$ & $(25)(134)$ & $(3)(2)(145)$ & $(3)(5)(2)(14)$ \\
\hline$(2)(3)(5)(4)$ & $(23)(1)(45)$ & $(25)(14)$ & $(3)(2)(15)$ & $(3)(5)(2)(4)$ \\
\hline$(2)(3)(5)(4)(1)$ & $(23)(1)(5)$ & $(25)(14)(3)$ & $(3)(2)(15)(4)$ & $(3)(5)(2)(4)(1)$ \\
\hline (2) (34) & $(23)(1)(5)(4)$ & $(25)(3)$ & $(3)(2)(4)$ & $(3)(5)(24)$ \\
\hline$(2)(34)(1)$ & $(23)(14)$ & $(25)(3)(1)$ & $(3)(2)(4)(1)$ & $(3)(5)(24)(1)$ \\
\hline$(2)(34)(1)(5)$ & $(23)(14)(5)$ & $(25)(3)(1)(4)$ & $(3)(2)(4)(1)(5)$ & $(3)(5)(4)$ \\
\hline$(2)(34)(15)$ & $(23)(145)$ & $(25)(3)(14)$ & $(3)(2)(4)(15)$ & $(3)(5)(4)(1)$ \\
\hline$(2)(34)(5)$ & $(23)(15)$ & $(25)(3)(4)$ & $(3)(2)(4)(5)$ & $(3)(5)(4)(1)(2)$ \\
\hline$(2)(34)(5)(1)$ & $(23)(15)(4)$ & $(25)(3)(4)(1)$ & $(3)(2)(4)(5)(1)$ & $(3)(5)(4)(12)$ \\
\hline (2) (345) & $(23)(4)$ & $(25)(34)$ & (3) (2) (45) & $(3)(5)(4)(2)$ \\
\hline$(2)(345)(1)$ & $(23)(4)(1)$ & $(25)(34)(1)$ & $(3)(2)(45)(1)$ & $(3)(5)(4)(2)(1)$ \\
\hline (2)(35) & $(23)(4)(1)(5)$ & $(25)(4)$ & $(3)(2)(5)$ & (34) \\
\hline (2)(35)(1) & $(23)(4)(15)$ & $(25)(4)(1)$ & $(3)(2)(5)(1)$ & $(34)(1)$ \\
\hline$(2)(35)(1)(4)$ & $(23)(4)(5)$ & $(25)(4)(1)(3)$ & $(3)(2)(5)(1)(4)$ & $(34)(1)(2)$ \\
\hline$(2)(35)(14)$ & $(23)(4)(5)(1)$ & $(25)(4)(13)$ & $(3)(2)(5)(14)$ & $(34)(1)(2)(5)$ \\
\hline$(2)(35)(4)$ & $(23)(45)$ & $(25)(4)(3)$ & $(3)(2)(5)(4)$ & $(34)(1)(25)$ \\
\hline$(2)(35)(4)(1)$ & $(23)(45)(1)$ & $(25)(4)(3)(1)$ & $(3)(2)(5)(4)(1)$ & $(34)(1)(5)$ \\
\hline$(2)(4)$ & $(23)(5)$ & (3) & (3) $(24)$ & $(34)(1)(5)(2)$ \\
\hline$(2)(4)(1)$ & $(23)(5)(1)$ & (3) (1) & $(3)(24)(1)$ & $(34)(12)$ \\
\hline$(2)(4)(1)(3)$ & $(23)(5)(1)(4)$ & (3) (1) (2) & $(3)(24)(1)(5)$ & $(34)(12)(5)$ \\
\hline$(2)(4)(1)(3)(5)$ & $(23)(5)(14)$ & $(3)(1)(2)(4)$ & $(3)(24)(15)$ & $(34)(125)$ \\
\hline$(2)(4)(1)(35)$ & $(23)(5)(4)$ & $(3)(1)(2)(4)(5)$ & $(3)(24)(5)$ & $(34)(15)$ \\
\hline$(2)(4)(1)(5)$ & $(23)(5)(4)(1)$ & (3) (1) (2) (45) & $(3)(24)(5)(1)$ & $(34)(15)(2)$ \\
\hline$(2)(4)(1)(5)(3)$ & $(234)$ & $(3)(1)(2)(5)$ & (3) (245) & $(34)(2)$ \\
\hline$(2)(4)(13)$ & $(234)(1)$ & $(3)(1)(2)(5)(4)$ & $(3)(245)(1)$ & $(34)(2)(1)$ \\
\hline$(2)(4)(13)(5)$ & $(234)(1)(5)$ & $(3)(1)(24)$ & (3) (25) & $(34)(2)(1)(5)$ \\
\hline$(2)(4)(135)$ & $(234)(15)$ & $(3)(1)(24)(5)$ & $(3)(25)(1)$ & $(34)(2)(15)$ \\
\hline$(2)(4)(15)$ & $(234)(5)$ & $(3)(1)(245)$ & $(3)(25)(1)(4)$ & $(34)(2)(5)$ \\
\hline$(2)(4)(15)(3)$ & $(234)(5)(1)$ & $(3)(1)(25)$ & $(3)(25)(14)$ & $(34)(2)(5)(1)$ \\
\hline$(2)(4)(3)$ & $(2345)$ & $(3)(1)(25)(4)$ & (3) (25) (4) & $(34)(25)$ \\
\hline$(2)(4)(3)(1)$ & $(2345)(1)$ & $(3)(1)(4)$ & $(3)(25)(4)(1)$ & $(34)(25)(1)$ \\
\hline$(2)(4)(3)(1)(5)$ & $(235)$ & $(3)(1)(4)(2)$ & (3) (4) & $(34)(5)$ \\
\hline$(2)(4)(3)(15)$ & $(235)(1)$ & $(3)(1)(4)(2)(5)$ & $(3)(4)(1)$ & $(34)(5)(1)$ \\
\hline$(2)(4)(3)(5)$ & $(235)(1)(4)$ & $(3)(1)(4)(25)$ & $(3)(4)(1)(2)$ & $(34)(5)(1)(2)$ \\
\hline$(2)(4)(3)(5)(1)$ & $(235)(14)$ & $(3)(1)(4)(5)$ & $(3)(4)(1)(2)(5)$ & $(34)(5)(12)$ \\
\hline$(2)(4)(35)$ & $(235)(4)$ & $(3)(1)(4)(5)(2)$ & $(3)(4)(1)(25)$ & $(34)(5)(2)$ \\
\hline$(2)(4)(35)(1)$ & $(235)(4)(1)$ & (3) (1) (45) & $(3)(4)(1)(5)$ & $(34)(5)(2)(1)$ \\
\hline$(2)(4)(5)$ & $(24)$ & $(3)(1)(45)(2)$ & $(3)(4)(1)(5)(2)$ & $(345)$ \\
\hline$(2)(4)(5)(1)$ & $(24)(1)$ & $(3)(1)(5)$ & $(3)(4)(12)$ & $(345)(1)$ \\
\hline$(2)(4)(5)(1)(3)$ & $(24)(1)(3)$ & $(3)(1)(5)(2)$ & $(3)(4)(12)(5)$ & $(345)(1)(2)$ \\
\hline$(2)(4)(5)(13)$ & $(24)(1)(3)(5)$ & $(3)(1)(5)(2)(4)$ & $(3)(4)(125)$ & $(345)(12)$ \\
\hline$(2)(4)(5)(3)$ & $(24)(1)(35)$ & $(3)(1)(5)(24)$ & $(3)(4)(15)$ & $(345)(2)$ \\
\hline$(2)(4)(5)(3)(1)$ & $(24)(1)(5)$ & $(3)(1)(5)(4)$ & $(3)(4)(15)(2)$ & $(345)(2)(1)$ \\
\hline (2) (45) & $(24)(1)(5)(3)$ & $(3)(1)(5)(4)(2)$ & $(3)(4)(2)$ & (35) \\
\hline$(2)(45)(1)$ & $(24)(13)$ & $(3)(12)$ & $(3)(4)(2)(1)$ & $(35)(1)$ \\
\hline$(2)(45)(1)(3)$ & $(24)(13)(5)$ & $(3)(12)(4)$ & $(3)(4)(2)(1)(5)$ & $(35)(1)(2)$ \\
\hline$(2)(45)(13)$ & $(24)(135)$ & $(3)(12)(4)(5)$ & $(3)(4)(2)(15)$ & $(35)(1)(2)(4)$ \\
\hline$(2)(45)(3)$ & $(24)(15)$ & $(3)(12)(45)$ & $(3)(4)(2)(5)$ & $(35)(1)(24)$ \\
\hline$(2)(45)(3)(1)$ & $(24)(15)(3)$ & $(3)(12)(5)$ & $(3)(4)(2)(5)(1)$ & $(35)(1)(4)$ \\
\hline (2)(5) & $(24)(3)$ & $(3)(12)(5)(4)$ & $(3)(4)(25)$ & $(35)(1)(4)(2)$ \\
\hline$(2)(5)(1)$ & $(24)(3)(1)$ & (3) (124) & $(3)(4)(25)(1)$ & $(35)(12)$ \\
\hline$(2)(5)(1)(3)$ & $(24)(3)(1)(5)$ & $(3)(124)(5)$ & $(3)(4)(5)$ & $(35)(12)(4)$ \\
\hline$(2)(5)(1)(3)(4)$ & $(24)(3)(15)$ & (3) (1245) & $(3)(4)(5)(1)$ & $(35)(124)$ \\
\hline$(2)(5)(1)(34)$ & $(24)(3)(5)$ & (3) (125) & $(3)(4)(5)(1)(2)$ & $(35)(14)$ \\
\hline$(2)(5)(1)(4)$ & $(24)(3)(5)(1)$ & $(3)(125)(4)$ & $(3)(4)(5)(12)$ & $(35)(14)(2)$ \\
\hline$(2)(5)(1)(4)(3)$ & $(24)(35)$ & (3) (14) & $(3)(4)(5)(2)$ & $(35)(2)$ \\
\hline$(2)(5)(13)$ & $(24)(35)(1)$ & (3) (14)(2) & $(3)(4)(5)(2)(1)$ & $(35)(2)(1)$ \\
\hline$(2)(5)(13)(4)$ & $(24)(5)$ & $(3)(14)(2)(5)$ & (3) (45) & $(35)(2)(1)(4)$ \\
\hline$(2)(5)(134)$ & $(24)(5)(1)$ & $(3)(14)(25)$ & $(3)(45)(1)$ & $(35)(2)(14)$ \\
\hline$(2)(5)(14)$ & $(24)(5)(1)(3)$ & $(3)(14)(5)$ & $(3)(45)(1)(2)$ & $(35)(2)(4)$ \\
\hline$(2)(5)(14)(3)$ & $(24)(5)(13)$ & $(3)(14)(5)(2)$ & $(3)(45)(12)$ & $(35)(2)(4)(1)$ \\
\hline$(2)(5)(3)$ & $(24)(5)(3)$ & $(3)(145)$ & $(3)(45)(2)$ & $(35)(24)$ \\
\hline$(2)(5)(3)(1)$ & $(24)(5)(3)(1)$ & $(3)(145)(2)$ & $(3)(45)(2)(1)$ & $(35)(24)(1)$ \\
\hline
\end{tabular}




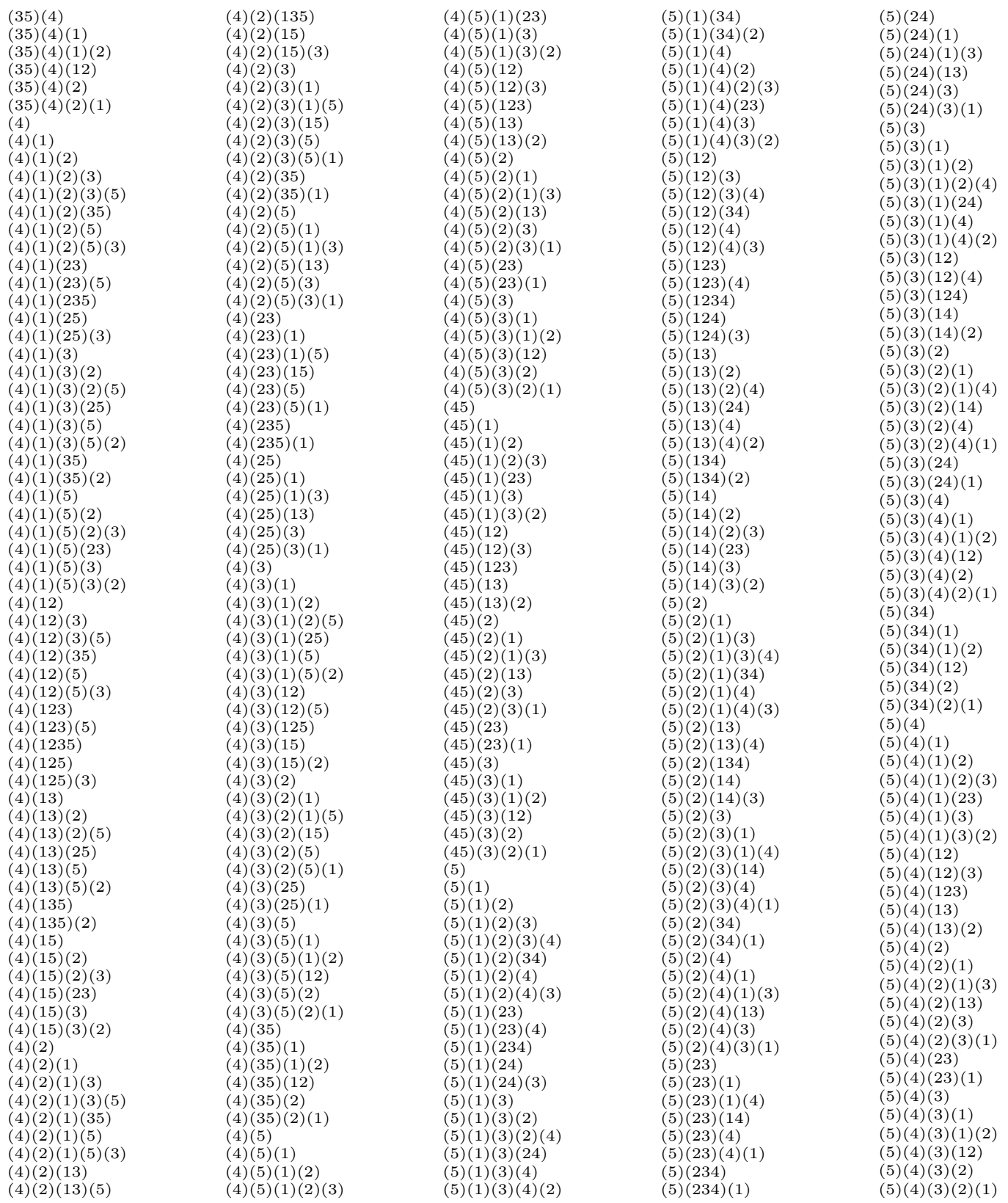




\section{The Five-Button Door Lock - Experiment And Discovery in Mathematics}

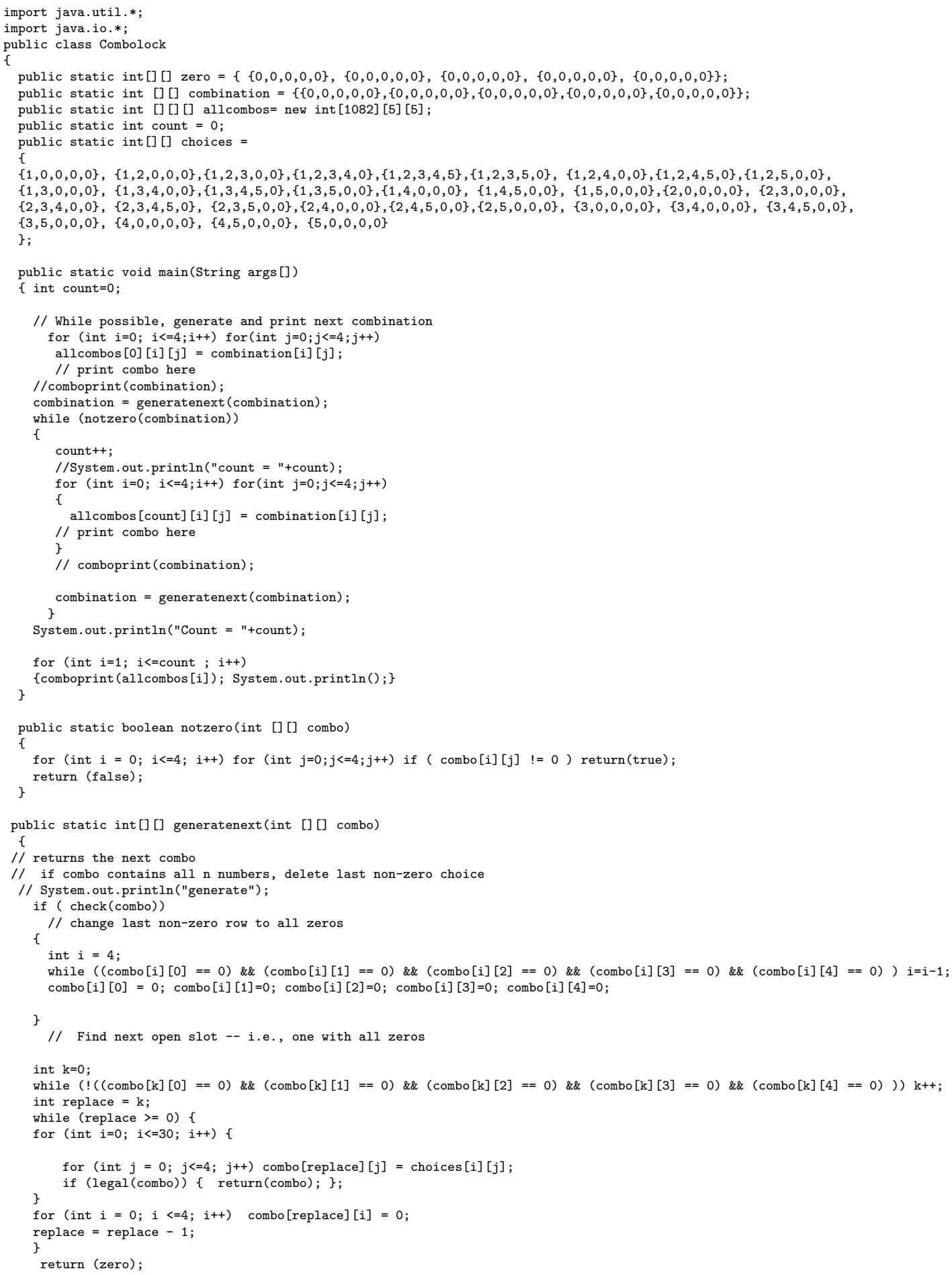




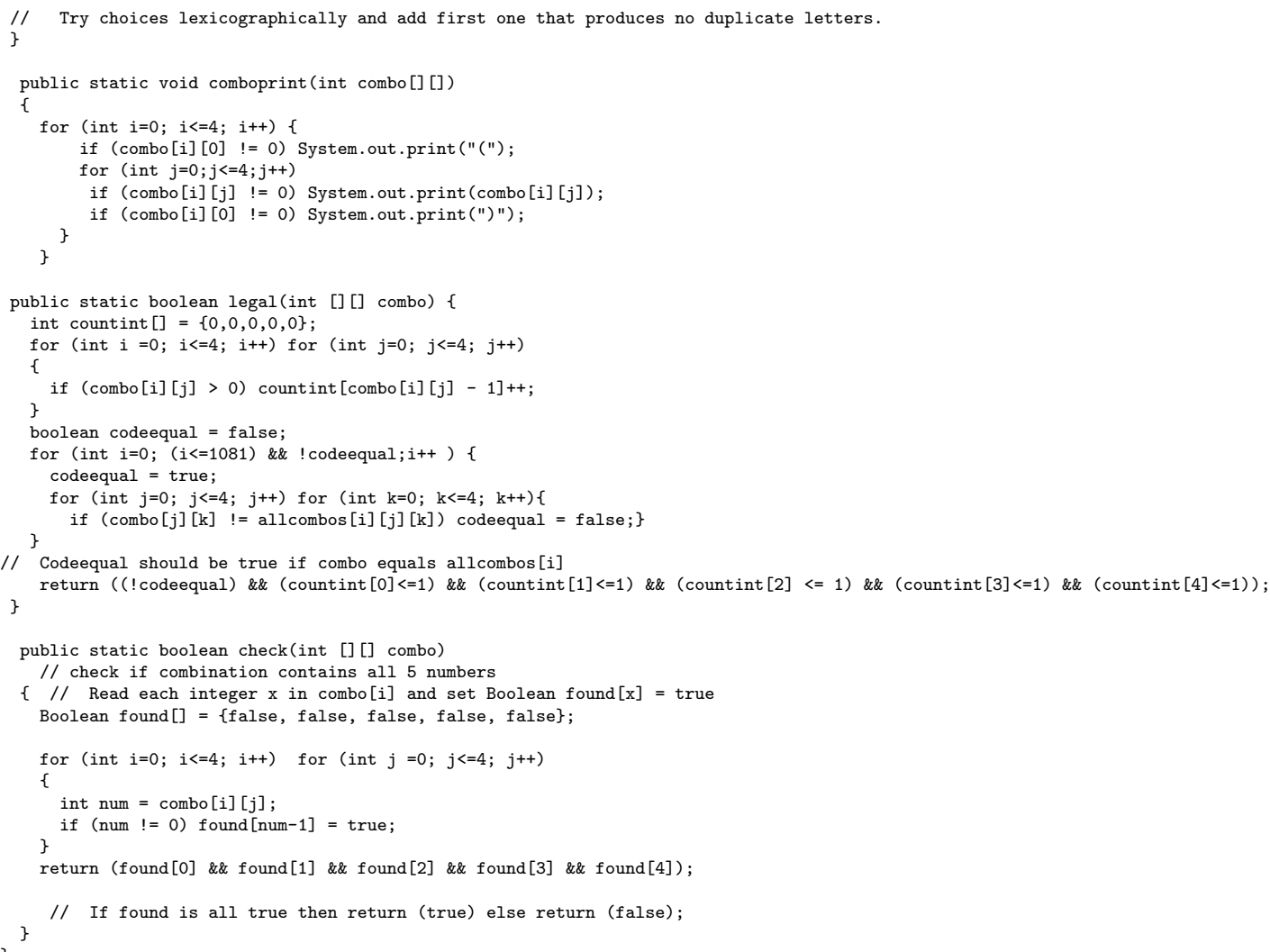

